\title{
The Comparison of Canopy Height Profiles Extracted from Ku-band Profile Radar Waveforms and LiDAR Data
}

\author{
Hui Zhou ${ }^{1,2}$, Yuwei Chen ${ }^{2, *}$, Ziyi Feng ${ }^{2}$, Fashuai Li ${ }^{2}{ }^{\mathbb{D}}$, Juha Hyyppä ${ }^{2}$, Teemu Hakala ${ }^{2}$, \\ Mika Karjalainen ${ }^{2}$, Changhui Jiang ${ }^{2,3}$ and Ling Pei ${ }^{4}$ \\ 1 Electronic Information School, Wuhan University, Wuhan 430079, China; zhouhui@whu.edu.cn \\ 2 Department of Remote Sensing and Photogrammetry, Finnish Geospatial Research Institute, Kirkkonummi \\ FI-02431, Finland; Ziyi.feng@nls.fi (Z.F.); f.li@utwente.nl (F.L.); juha.hyyppa@nls.fi (J.H.); \\ teemu.hakala@nls.fi (T.H.); mika.kirjalainen@nls.fi (M.K.); chiang_changhui@outlook.coom (C.J.) \\ 3 School of automation, Nanjing University of Science and Technology, Nanjing 210094, China \\ 4 Shanghai Key Laboratory of Navigation and Location-based Services, School of Electronic Information and \\ Electrical Engineering, Shanghai Jiao Tong University, Shanghai 200241, China; ling.pei@sjtu.edu.cn \\ * Correspondence: Yuwei.chen@nls.fi; Tel.: +358-40-703-9098
}

Received: 5 March 2018; Accepted: 25 April 2018; Published: 3 May 2018

\begin{abstract}
An airborne Ku-band frequency-modulated continuous waveform (FM-CW) profiling radar, Tomoradar, records the backscatter signal from the canopy surface and the underlying ground in the southern boreal forest zone of Finland. The recorded waveforms are transformed into canopy height profiles (CHP) with a similar methodology utilized in large-footprint light detection and ranging (LiDAR). The point cloud data simultaneously collected by a Velodyne ${ }^{\circledR}$ VLP-16 LiDAR on-board the same platform represent the frequency of discrete returns, which are also applied to the extraction of the CHP by calculating the gap probability and incremental distribution. To thoroughly explore the relationships of the CHP derived from Tomoradar waveforms and LiDAR data we utilized the effective waveforms of one-stripe field measurements and comparison them with four indicators, including the correlation coefficient, the root-mean-square error (RMSE) of the difference, and the coefficient of determination and the RMSE of residuals of linear regression. By setting the Tomoradar footprint as 20 degrees to contain over $95 \%$ of the transmitting energy of the main lobe, the results show that $88.17 \%$ of the CHPs derived from Tomoradar waveforms correlated well with those from the LiDAR data; $98 \%$ of the RMSEs of the difference ranged between 0.002 and $0.01 ; 79.89 \%$ of the coefficients of determination were larger than 0.5 ; and $98.89 \%$ of the RMSEs of the residuals ranged from 0.001 to 0.01 . Based on the investigations, we discovered that the locations of the greatest CHP derived from the Tomoradar were obviously deeper than those from the LiDAR, which indicated that the Tomoradar microwave signal had a stronger penetration capability than the LiDAR signal. Meanwhile, there are smaller differences (the average RMSEs of differences is only 0.0042 when the total canopy closure is less than 0.5 ) and better linear regression results in an area with a relatively open canopy than with a denser canopy.
\end{abstract}

Keywords: Ku band profile radar; LiDAR; power waveform; point cloud; canopy height profile

\section{Introduction}

Forest covers approximate $30 \%$ of the global land area, which plays a significant role in the natural circulation of carbon and the mitigation of climate change [1]. These important ecological processes are usually affected by the canopy vertical structure (CVS), which is defined as the quantity and arrangement of the canopy along the vertical axis [2]. As a characterization of CVS, the canopy 
height profile (CHP) represents the relative canopy vertical distribution that depicts the fraction of total plant area.

There are two categories of measurement techniques to estimate the CHP: traditional ground methods and advanced remote sensing approaches. A representative ground method to obtain canopy height profiles is the point sampling quadrat provided by Wilson, who measured the points of intercepting foliage along a series of vertical transects from the top to bottom of the canopy by utilizing several lines [3]. An improved point quadrat method was developed by MacArthur and Horn using a calibrated telephoto camera [4], and applied by Arber to northern temperate canopies [5]. These field inventories are obviously time-consuming, laborious, expensive, and inappropriate for a large-scale measurement.

Conversely, remote sensing technology can provide more accurate measurement of spatially-continuous properties of forest structure over large areas in a rapid manner. Passive optical remote sensing systems mainly collect the reflected sunlight from the exterior canopy surface and are almost unable to acquire the information inside the canopy due to topographical occlusion, the limitation of the employed spectral range, and poor illustration conditions. They can hardly capture the vertical forest structure directly and exclusively obtain the limited characterization of the canopy structure, such as tree crown and leaf area index (LAI) [6-8]. Airborne light detection and ranging (LiDAR) can partially penetrate the canopy and reveal the vertical structure of the canopy by using small-footprint laser data, such as discrete returns or digitalized waveforms [9]. There are two typical processing approaches to extract the CHP with small-footprint LiDAR data: (1) derivation of gap probability and the corresponding incremental distribution at optional areas for LiDAR point clouds with high point densities [10-13]; and (2) the methodology used in spaceborne large-footprint full waveform LiDAR [14,15], but small-footprint LiDAR waveforms first need to be aligned according to the elevation and summed to generate an accumulative large-footprint waveform [16-18]. According to the character of full waveforms and their relationships with CVS, Harding et al. [15] provided the procedures of transforming waveform data of the Scanning LiDAR Imager of Canopies by Echo Recovery (SLICER) into the CHP and validated the results with the ground-based measurements.

For acquiring canopy height and other vertical canopy structure information, a light-weighted Ku-band frequency-modulated continuous waveform (FM-CW) airborne profiling radar, named Tomoradar, was designed by the Finnish Geospatial Research Institute (FGI), which is currently the only available Ku-band profiling radar for forest measurements to the best of our knowledge [19-21]. Tomoradar on-board an airborne platform can collect the full polarization backscattered waveforms within the footprint of several meters from the forest with an operational altitude of several tens of meters. Furthermore, a Velodyne ${ }^{\circledR}$ VLP-16 LiDAR installed on the same platform was employed to offer a coincident laser point cloud. Considerable amount of research has been carried out in the field of retrieving forest biomass from satellite radar data. There have been two main research lines, using either (1) SAR backscattering information [22] or (2) elevation models extracted from the SAR data [23], to retrieve forest biomass estimation models. Without going too deep into the details of these techniques, one of the main challenges today is to better understand the interaction of radar waves with forest vertical structures under variable environmental conditions. By comparing the Tomoradar waveforms and high-density ALS data, it may be possible to advance the understanding of spaceborne radar responses from forests as well and, accordingly, contribute to the current SAR satellite-based biomass retrieval models. Thus, in this paper, the estimations of the CHP, which is a typical characterization parameter, are investigated using Tomoradar waveforms and LiDAR discrete returns.

In this research, a processing method of deriving the CHP from Tomoradar waveforms was developed on the basis of the methodology used in the large-footprint waveform LiDAR while, for LiDAR data, the CHP extracted from the small-footprint LiDAR with discrete returns were utilized. One stripe of the Tomoradar field measurements containing 6766 effective waveforms of boreal forest data in Southern Finland were employed to extract the canopy height profile. According to the 
difference and linear regression analysis, the comparisons of CHP extracted from Tomoradar and LiDAR were investigated thoroughly. The results may provide guidance for potential applications of using radar sensors for forest inventories.

The rest of this paper is organized as follows: Section 2 describes the study area, Tomoradar waveforms, and LiDAR data, and illustrates the methods of derivation and comparisons of the CHP; Section 3 expounds the comparison of CHP result; and, finally, the conclusions are drawn in Section 4.

\section{Materials and Methods}

\subsection{Site Description}

The study area for acquiring the field data on 21 September 2016 was located at Evo in Southern Finland $\left(61^{\circ} 19^{\prime} \mathrm{N}, 25^{\circ} 11^{\prime} \mathrm{E}\right)$. It is a part of the southern boreal forest zone and consists predominantly of Scots pine, Norway spruce, and birch. Distinguished from a completely homogenous managed forest, this site is a popular recreation area and contributes to the analysis of the Tomoradar waveforms from different vegetation structures, including the composition, geometry, roughness, and density of canopy components. The test site is composed of three sections: the east section, west section, and south section, and experimental data investigated in this paper are chosen from one stripe in the west section (Figure 1a). Both the Tomoradar developed by FGI and VLP-16 LiDAR manufactured by Velodyne, USA were installed on an extended arm of a Bell 206 helicopter, with a flight height of 60-100 m and a flight velocity of 10-20 m/s. Meanwhile, a Novatel tightly-coupled positioning system consisting of a Global Navigation Satellite System and an inertial measurement unit (GNSS-IMU) attached on the frame offered centimeter-level accuracy trajectory information of the helicopter, which was triggered by internal TTL signals sent by the Tomoradar. Thus, the Tomoradar measurements were time-tagged with GPS time, and the LiDAR was synchronized to the GPS time with an extra GNSS receiver. As a result, both the Tomoradar data and LiDAR can be aligned in global coordinates, and both sets of data (Velodyne ${ }^{\circledR}$ LiDAR-Tomoradar) can be overlapped at the centimeter-level. The configurations of all instruments on the helicopter are illustrated in Figure $1 \mathrm{~b}$.

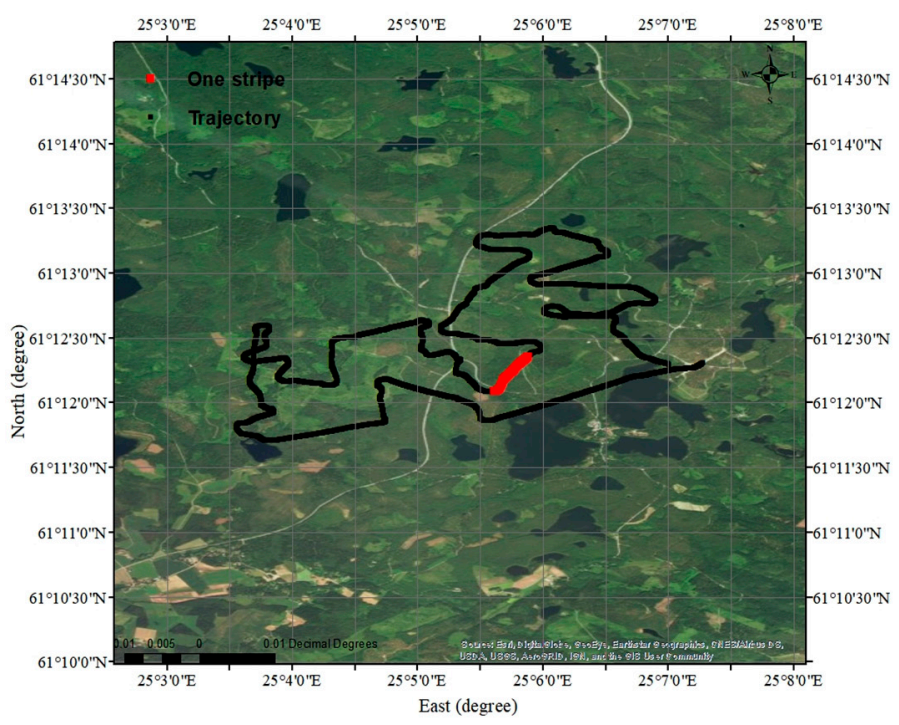

(a)

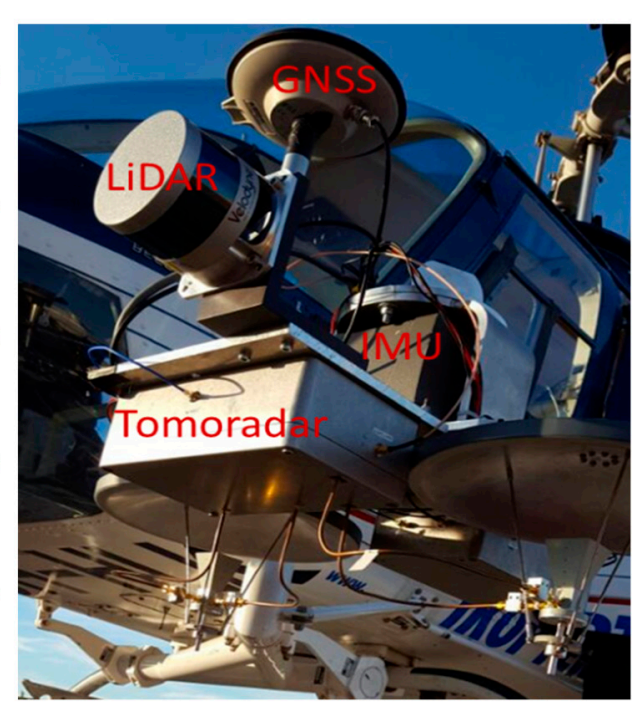

(b)

Figure 1. (a) Trajectory diagram in the west section of study area (black line), and a route of investigated data in one stripe (red line); and (b) the configurations of the instruments on the Bell 206 helicopter: Tomoradar, LiDAR, and GNSS-IMU. 


\subsection{Tomoradar Waveforms}

The FM-CW Tomoradar operating in the $\mathrm{Ku}$ band with a centre frequency $14 \mathrm{GHz}$ and a bandwidth of $1 \mathrm{GHz}$ transmits microwave radiation with a divergence of six degrees $(-3 \mathrm{~dB})$ and receives backscattered signals reflected from measured targets within the Tomoradar footprint [24]. The Tomoradar receiver is capable of receiving signals in four polarization modes (VV, VH, $\mathrm{HV}$, and $\mathrm{HH})$ with a $163 \mathrm{~Hz}$ modulation frequency, and converting them into waveforms with a $15 \mathrm{~cm}$ bin range resolution by its internal amplifier and digitizer.

Raw Tomoradar waveforms include the intensity/amplitude of backscattered microwave signal strength expressed in volt units as a function of distance. However, the CHP are derived according to the characteristics of power signals, which implies that raw Tomoradar waveforms need to be transformed to power waveforms. About 10,002 co-polarization $(\mathrm{HH})$ Tomoradar measurements from one stripe with an along-track distance of approximately $600 \mathrm{~m}$ were utilized to extract the canopy height profile, and raw waveforms and converted power waveforms are presented in Figure 2a,b. In order to interpret the distribution of the power waveform, we selected a single profile measurement at the green line marked location of the power waveforms, as illustrated in Figure 2c.

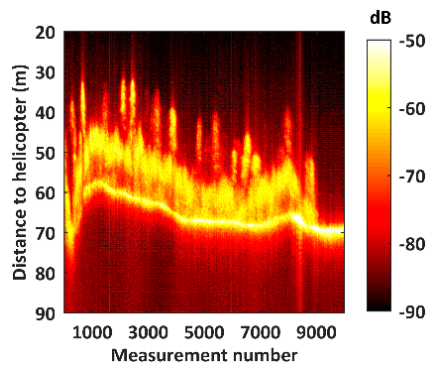

(a)

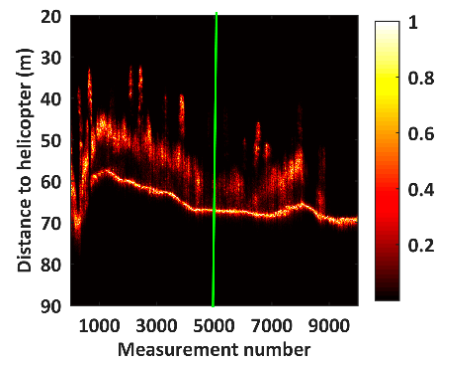

(b)

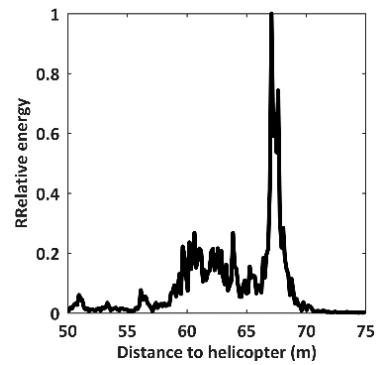

(c)

Figure 2. (a) Raw Tomoradar waveform profiles in one stripe in $\mathrm{HH}$ mode; (b) normalized power waveforms converted from raw Tomoradar waveforms; (c) a single power waveform at the green line location of the power waveforms. All raw Tomoradar waveforms are scaled from $20 \mathrm{~m}$ to $90 \mathrm{~m}$ for better illustration, even though they were recorded from $10 \mathrm{~m}$ to $150 \mathrm{~m}$. The power waveform is normalized by its corresponding maximum of each measurement.

In Figure 2, we observed that the normalized power waveforms are essentially similar to the raw Tomoradar waveforms, but include lower noise levels. The single power waveform can be treated as a distance-resolved measure of microwave radiation backscattered from the canopy surface to the underlying ground. These power waveforms were employed to extract the canopy height profile for this research.

\subsection{LiDAR Data}

The Velodyne ${ }^{\circledR}$ VLP-16 laser scanner operating with 300,000 points/second was installed strictly on the same platform as the Tomoradar to offer a laser point cloud of the study area. The Velodyne ${ }^{\circledR}$ LiDAR records the first and strongest return for every transmitted laser pulse with a beam size of $12.7 \mathrm{~mm}$ (horizontal) $\times 9.5 \mathrm{~mm}$ (vertical) at the exit with a beam divergence of approximately three milliradians [25], and instantaneously provides 16 laser footprints with an interval of two degrees on the ground along the flying trajectory. With a rotation of 360 degrees of the laser scanner, 16 parallel scan lines are simultaneously generated with uniform distributions across the flying track. The average point density was approximately 36 points per square meter for the selected stripe. For the purpose of determining the centres of the laser point cloud within the Tomoradar footprint on the ground, higher-precision LiDAR data collected by a Leica ${ }^{\circledR}$ ALS70-HA laser scanner at Evo in 2015 were also utilized. However, the data is mainly used as a DEM model, and all the comparisons were based on data collected by VLP-16 laser scanner and Tomoradar. 


\subsection{Methods}

\subsubsection{Derivation of Canopy Height Profile from Tomoradar Waveforms}

Tomoradar power waveforms representing the magnitudes of the backscattered power signal at different distances have basically identical connotation with full waveforms of a large-footprint LiDAR. According to the methodology used in the SLICER canopy height profile presented by Harding et al. [15], we assumed a horizontally random distribution of the canopy components and then estimated a CHP based on Tomoradar power waveforms by implementing a series of processing steps shown in Figure 3. The corresponding illustration of converting the Tomoradar waveform into the CHP is elaborated in Figure 4: (a) original waveform; (b) filtering noise, identifying the canopy and the ground; (c) calculating the canopy closure profile, transforming it into cumulative plant areas; and (d) yielding an incremental distribution (CHP).

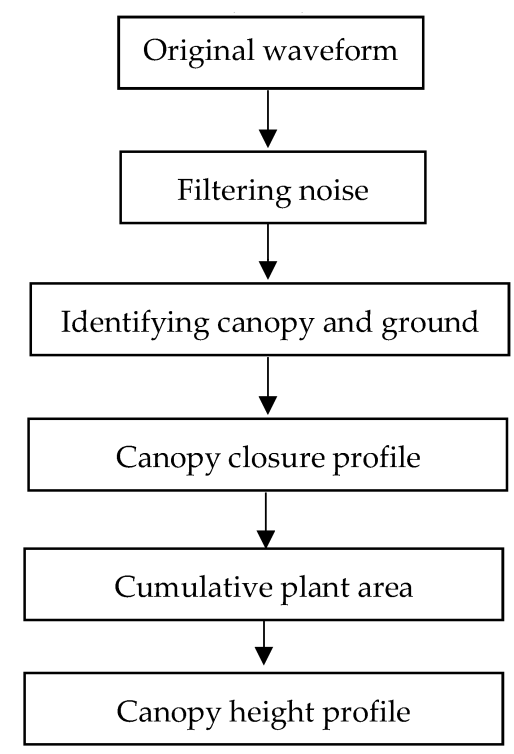

Figure 3. The flowchart of deriving the CHP from Tomoradar waveforms.

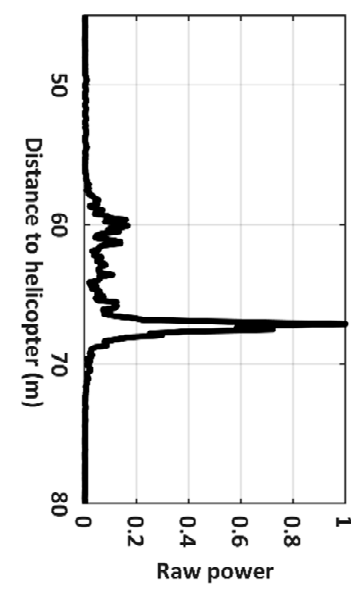

(a)

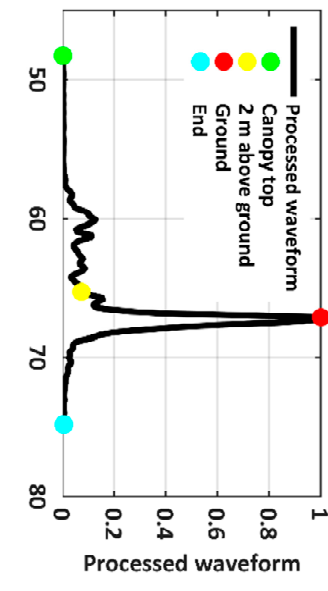

(b)

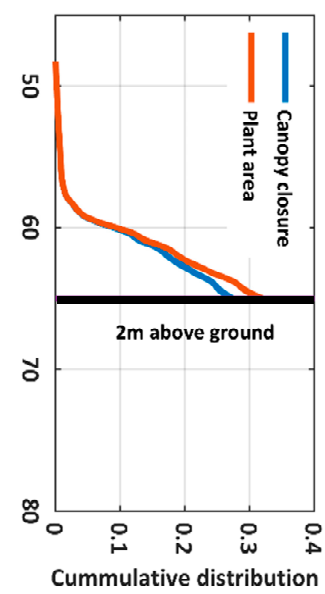

(c)

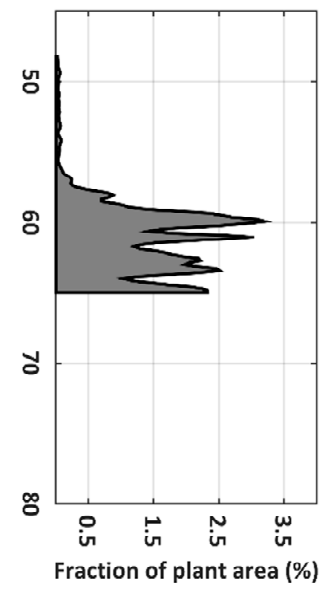

(d)

Figure 4. The processing steps of converting Tomoradar waveforms into the CHP: (a) raw power waveform with $15 \mathrm{~cm}$ range resolution; (b) processed waveforms by filtering noise, and the identified canopy top, $2 \mathrm{~m}$ above ground, center, and end of the ground; (c) the canopy closure profile and cumulative plant area; and (d) the incremental distribution of the plant area $(\mathrm{CHP})$ at $2 \mathrm{~m}$ above ground. 
(1) Filtering Noise for Raw Power Waveform

In order to eliminate the influence of noise on the power signal and improve the signal to noise ratio of the power waveform, a weighted averaging factor with a normalized Gaussian distribution $f(d)$ was convolved with the raw power waveform $P_{r}(d)$ [26], and the processed power waveform $P_{f}(d)$ was described as:

$$
P_{f}(d)=\sum_{k} P_{r}\left(d_{k}\right) f\left(d-d_{k}+\Delta d\right), \text { and } f(d)=\frac{1}{\sqrt{2 \pi} \omega} \exp \left(-\frac{d^{2}}{2 \omega^{2}}\right)
$$

Here, $\Delta d$ is the sampling resolution of the Tomoradar waveform, $d_{k}$ takes values from $-3 \omega$ to $3 \omega$, where $\omega$ is the root mean square (RMS) width of the Gaussian function. The filtering window seven times the length of the signals is employed to mitigate the noise level and smooth the raw power waveform.

\section{(2) Identifying Canopy and Ground}

The last return above noise is supposed to be the backscattered signal from the ground, and its peak location can be determined by searching for the last one of local maximum points of the processed power waveform. We approximately considered the position as the centroid of the Tomoradar footprint on the ground, and selected a point $2 \mathrm{~m}$ above ground as a boundary to differentiate the ground return and canopy return in this paper. The canopy top and end of the ground were identified by seeking the first and latest signal above a threshold that was three times the standard deviation of the noise according to three-standard-deviations principle.

\section{(3) Canopy Closure Profile}

A return energy profile $\mathrm{E}(\mathrm{d})$ was produced through calculating the area underneath each processed power waveform. The energy within each sampling interval roughly is the average of adjacent power amplitudes multiplied by the time interval represented by the $15 \mathrm{~cm}$ range resolution. Then a cumulative height distribution of the canopy return (canopy closure profile) was computed by sequentially adding up the energy profile from the canopy top to $2 \mathrm{~m}$ above ground, and normalized by the total energy including the canopy return and ground return. Considering the difference of the scattering coefficient between the canopy and the ground, the canopy closure profile $C(d)$ was adjusted by scaling the ground energy as [27]:

$$
C\left(d_{i}\right)=\frac{\sum_{k=t o p}^{i+1} E\left(d_{k}\right)}{\sum_{k=t o p}^{2 m} E\left(d_{k}\right)+\frac{1}{\gamma} \sum_{k=2 m}^{e n d} E\left(d_{k}\right)} \text {, and } d_{c} \leq d_{i} \leq d_{2}
$$

where $d_{i}$ and $d_{k}$ denote the distance from the helicopter to the $i$-th and $k$-th canopy layer; $d_{c}$ and $d_{2}$ are the distances from the helicopter to the canopy top and the $2 \mathrm{~m}$ boundary above ground, respectively. The scaling factor $\gamma$, corresponding to scattering coefficient ratio between canopy and ground, is supposed to be one in the paper, since the reflectance ratio is also ignored when calculating the canopy closure with discrete LiDAR returns in the following section.

The last element of the canopy closure profile (total canopy closure) is the ratio of the canopy returns to ground returns, and is closely relevant to the density of the canopy. A small total canopy closure indicates open canopy woodlands allowing more microwave energy to penetrate inside their canopy. Conversely, a large total canopy closure can be produced when the microwave projects into a relatively closed canopy. 
(4) Cumulative Distribution of the Plant Area

The consequence of occlusion of the canopy closure profile was corrected by applying the MacArthur-Horn methodology [3], similar to the processing approach of the LiDAR signal, and then transformed to the cumulative plant area $A_{T}\left(d_{i}\right)$ by the following:

$$
A_{T}\left(d_{i}\right)=-\ln \left[1-C\left(d_{i}\right)\right]
$$

We noticed that the cumulative plant area in Equation (3) is proportional to the canopy closure profile. Hence, the total received power of the Tomoradar from the canopy rises with the increase of the penetration depth into the canopy due to the effect of occlusion.

(5) The CHP Extracted from Tomoradar Waveforms

The CHP was yielded by converting the cumulative plant area into an incremental distribution and normalizing by the maximum of the cumulative plant area calculated by Equation (3), which can be expressed by:

$$
F_{T}\left(d_{i}\right)=\frac{\operatorname{diff}\left[A_{T}\left(d_{i}\right)\right]}{\max \left[A_{T}\left(d_{i}\right)\right]},
$$

where $\operatorname{diff}\left(A_{T}\right)$ denotes the differential of the cumulative plant area. The CHP $F_{T}\left(d_{i}\right)$ at different distances denotes the equivalent fraction of total plant area.

\subsubsection{Derivation of Canopy Height Profile from LiDAR Data}

As for the study stripe, the diameter of the Tomoradar footprint on the ground varied from a minimum of $6.1 \mathrm{~m}$ to a maximum of $7.4 \mathrm{~m}$ due to flying height changes between $57.4 \mathrm{~m}$ and $70.7 \mathrm{~m}$. Only the LiDAR data inside each Tomoradar footprint cone was accepted to be used to compute the CHP, as illustrated in Figure 5.

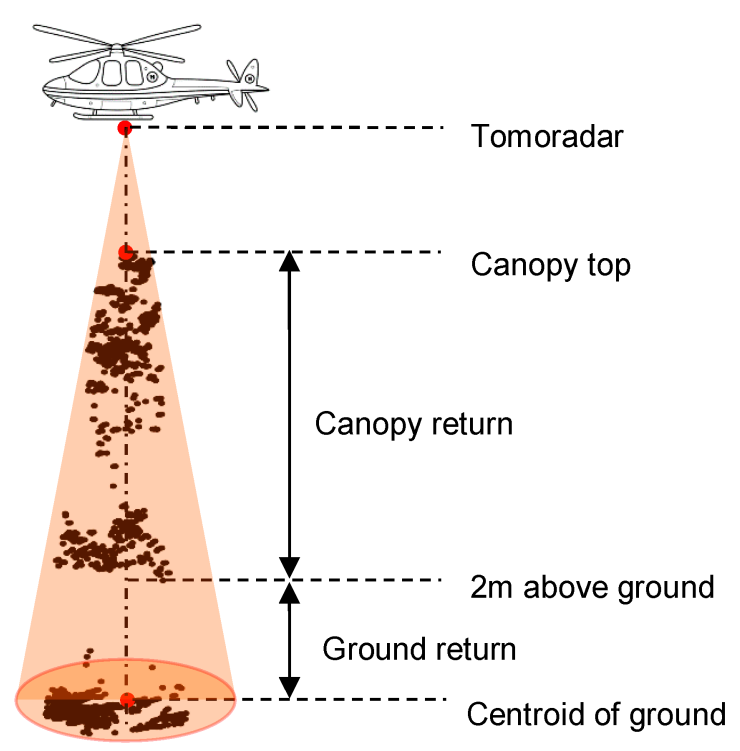

(a)

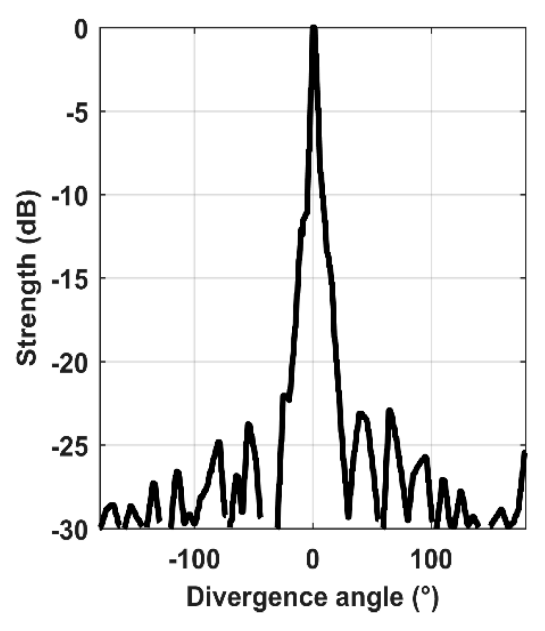

(b)

Figure 5. (a) The Velodyne ${ }^{\circledR}$ LiDAR point cloud within one Tomoradar footprint cone, the ground return and canopy return are derived by calculating the number of point cloud in the specified region; and (b) the Tomoradar transmitted antenna pattern, and its divergence angle is inconstant with the variability of strength. 
The gap probability $G_{p}\left(d_{i}\right)$ can be estimated by accumulating the numbers of laser hits from the canopy top to a certain distance $d$ and normalizing it by the total numbers of LiDAR point clouds $(N)$ within the Tomoradar footprint cone:

$$
G_{p}\left(d_{i}\right)=1-\frac{\left\{\# d_{k} \mid d_{k}>d_{i}\right\}}{N} .
$$

where $\# d_{k}$ represents the numbers of laser hits down to a preset boundary $d_{i}$ above the ground $(2 \mathrm{~m}$ in this research).

Likewise, we can also acquire the cumulative plant area $A_{L}\left(d_{i}\right)$ and the $\mathrm{CHP} F_{L}\left(d_{i}\right)$ derived from the LiDAR data based on the MacArthur-Horn methodology and the definition of CHP, which can be described by:

$$
A_{L}\left(d_{i}\right)=-\ln \left[G_{p}\left(d_{i}\right)\right], \text { and } F_{L}\left(d_{i}\right)=\frac{\operatorname{diff}\left(A_{L}\left(d_{i}\right)\right)}{\max \left(A_{L}\right)}
$$

\subsubsection{Comparison Method}

To describe the differences of the CHP extracted from Tomoradar waveforms $\left(F_{T}\right)$ and LiDAR data $\left(F_{L}\right)$, a correlation coefficient $\left(R^{2}\right)$ and a RMSE of the differences $\left(\delta_{d}\right)$ were computed by the following expressions:

$$
R^{2}=\frac{\sum\left(F_{T}-\overline{F_{T}}\right)\left(F_{L}-\overline{F_{L}}\right)}{\sqrt{\sum\left(F_{T}-\overline{F_{T}}\right)^{2} \sum\left(F_{L}-\overline{F_{L}}\right)^{2}}} \text {, and } \delta_{d}=\sqrt{\frac{\sum\left(F_{T}-F_{L}\right)^{2}}{n-1}}
$$

where $\overline{F_{T}}$ and $\overline{F_{L}}$ are the means of $F_{T}$ and $F_{L}$, and $n$ is the number of canopy height profiles.

Additionally, a linear regression analysis was applied in estimating the relationships among $F_{T}$ and $F_{L}$. If we define that $F_{R}$ is the fitted value of $F_{T}$ with a linear model, then the coefficient of determination $\left(r^{2}\right)$ and the RMSE of residuals $\left(\delta_{R}\right)$ are given by:

$$
r^{2}=1-\frac{\sum\left(F_{T}-F_{R}\right)^{2}}{\sum\left(F_{T}-\overline{F_{T}}\right)^{2}}, \text { and } \delta_{R}=\sqrt{\frac{\sum\left(F_{T}-F_{R}\right)^{2}}{n-1}}
$$

\section{Results and Discussion}

Due to the complexity of the flight trajectory, only Tomoradar measurements at the proximate nadir direction are applicable to derive the CHP. The nadir angle of the Tomoradar can be determined according to the roll angles from the IMU onboard the helicopter. When the nadir angles are less than five degrees, approximately $67 \%$ of the raw waveforms (6766 of 10,002 measurements) are reserved to be investigated.

According to above-mentioned method, we compute the CHP for 6766 measurements by using the Tomoradar waveforms and LiDAR data, the distributions of which versus the distances to the helicopter are illustrated in Figure 6.

It can be perceived that the tendency of the CHP extracted from Tomoradar waveforms are nearly similar as those from the LiDAR data, as Figure $6 \mathrm{a}, \mathrm{b}$ presents. From the canopy top to $2 \mathrm{~m}$ above ground, the CHP are multimodal structures for all the measurements, as an example of the 6000th measurement in Figure 6c. Moreover, the locations of greatest CHP in Figure 6d derived from Tomoradar results are obviously deeper than those from LiDAR, and the average difference approaches $2.59 \mathrm{~m}$. This demonstrates that more microwave energy passes through the interior of the canopy, which proves the Tomoradar microwave signal has a stronger penetration capability than the LiDAR signal even though the Ku band has lower penetration than longer-wavelength bands, such as the $\mathrm{C}$ band and P band. 


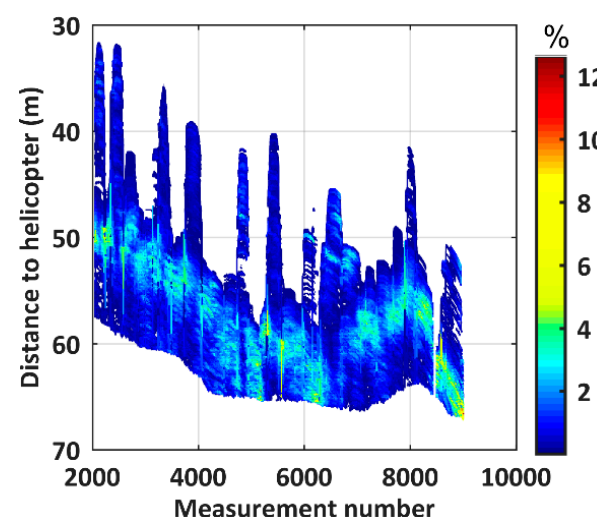

(a)

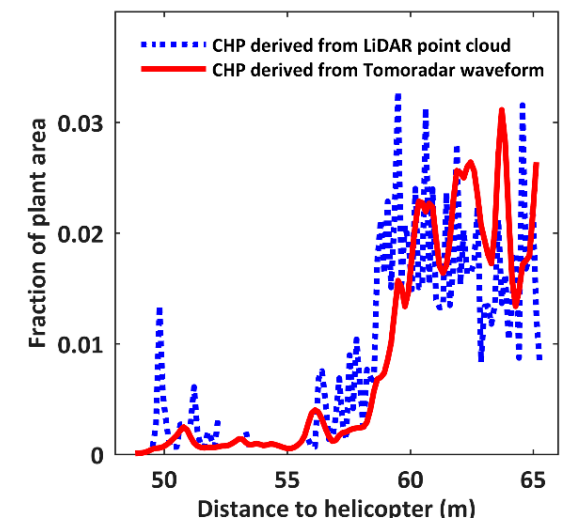

(c)

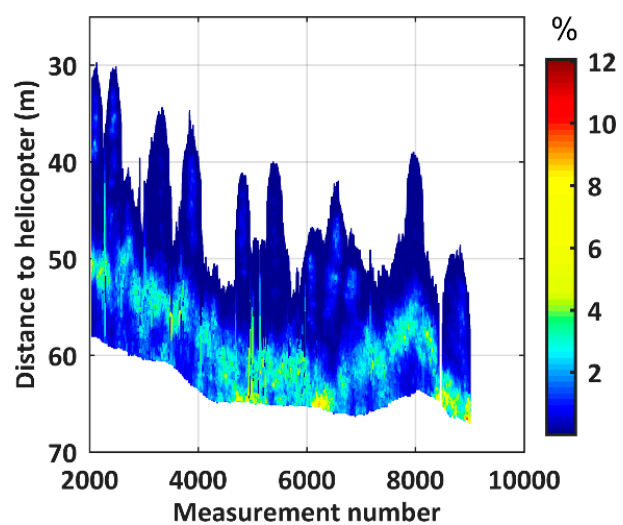

(b)

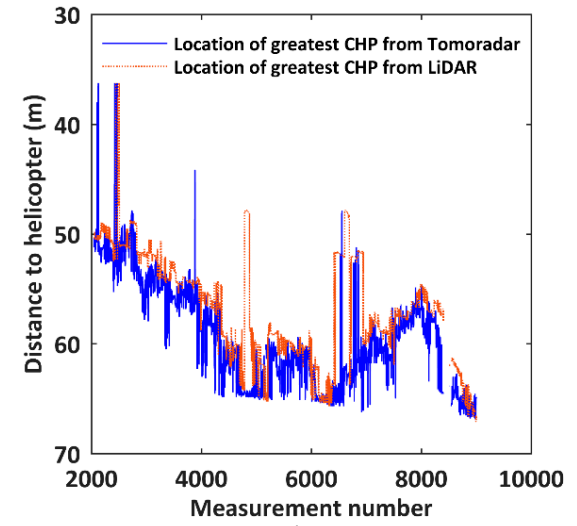

(d)

Figure 6. The distributions of CHP for 6676 measurements: (a) the CHPs from the LiDAR data; (b) the CHPs from Tomoradar waveforms; (c) the CHPs from Tomoradar and LiDAR for the 6000th measurement; and (d) the locations of greatest CHP.

\subsection{Analysis of the Differences of the CHPs}

To quantitatively compare the relationships of the CHPs extracted from Tomoradar and LiDAR data, we first calculate the correlation coefficients for all reserved measurements according to Equation (7) and present a histogram based on the strength of the correlation in Figure 7.

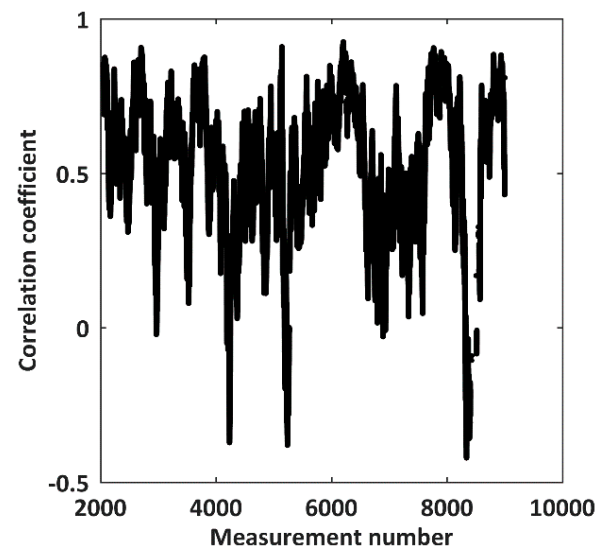

(a)

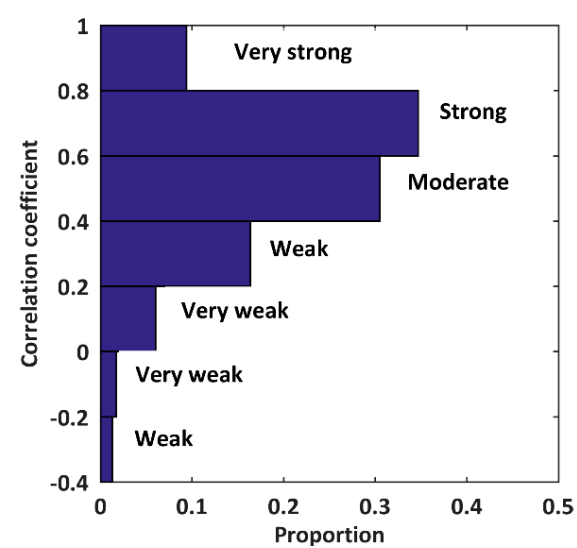

(b)

Figure 7. (a) The correlation coefficients of CHPs derived from Tomoradar and LiDAR; and (b) the proportions of correlation coefficients to 6766 measurements based on the strength of correlation. If a correlation coefficient is greater than zero, it is a positive correlation. On the contrary, it is a negative relationship. 
In Figure 7a, the correlation coefficients vary with the measurement number, and range between -0.40 and 0.93 . According to the correlation strength, we divide the correlation coefficients into 10 sections with an interval of 0.2 when they take values from -1 to 1 . By counting the numbers within each section, we obtain the corresponding proportions to all 6766 measurements (Figure 7b) and enumerate them in Table 1.

Table 1. The proportions of the CHP with different correlation strengths.

\begin{tabular}{ccccccc}
\hline $\begin{array}{c}\text { Weak and } \\
\text { Negative }\end{array}$ & $\begin{array}{c}\text { Very Weak } \\
\text { and Negative }\end{array}$ & $\begin{array}{c}\text { Very Weak } \\
\text { and Positive }\end{array}$ & $\begin{array}{c}\text { Weak and } \\
\text { Positive }\end{array}$ & $\begin{array}{c}\text { Moderate and } \\
\text { Positive }\end{array}$ & $\begin{array}{c}\text { Strong and } \\
\text { Positive }\end{array}$ & $\begin{array}{c}\text { Very Strong } \\
\text { and Positive }\end{array}$ \\
\hline $1.29 \%$ & $1.74 \%$ & $6.01 \%$ & $16.37 \%$ & $30.53 \%$ & $34.67 \%$ & $9.39 \%$ \\
\hline
\end{tabular}

Most of the CHPs (74.59\%) derived from Tomoradar and LiDAR data have above-moderate correlation strength, and only $25.41 \%$ of the CHPs correlate weakly or very weakly. To explore the outliers, we select the worst one (the 5270th measurement) with a correlation strength of zero and illustrate the matching Tomoradar waveform and LiDAR data in Figure 8.

The Tomoradar waveform at the 5270th measurement (Figure 8a) is correctly processed and four feature points, including the canopy top, $2 \mathrm{~m}$ above ground, centroid of ground, and end of ground, are precisely extracted, and the canopy height extracted from Figure 7a is approximately $20 \mathrm{~m}$. Consequently, the weak correlation strength is owning to the coincident LiDAR data (Figure 8b). We discover that the canopy height of the coincident LiDAR is about $10 \mathrm{~m}$ as the red-colored point cloud indicates, however, there are some higher trees with a canopy height of approximately $22 \mathrm{~m}$ in the neighboring area which are obviously different from the vegetation of the 5270th measurement. We believe that main lobe leakage should be the cause for such a phenomenon, which is similar to the canopy estimation outlier found in [28]. To verify their impacts on the extraction of the canopy height profile, the Tomoradar footprint cone is expanded from six degrees to 12 degrees on purpose to contain these trees. The CHP derived from the 5270th Tomoradar waveforms and LiDAR data within cones of six degrees and 12 degrees are shown in Figure 9.

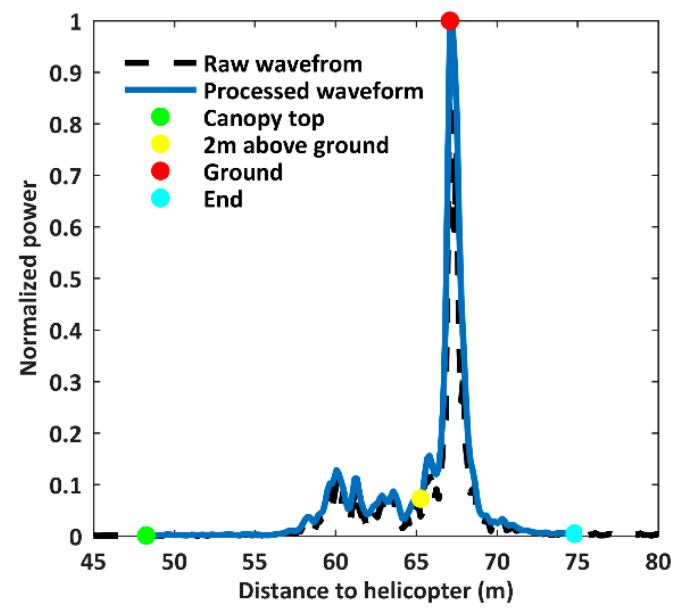

(a)

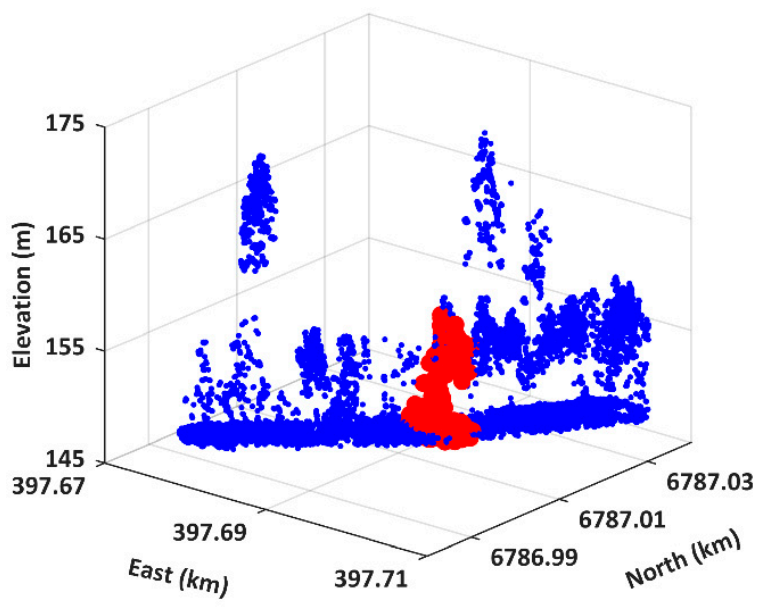

(b)

Figure 8. The Tomoradar waveform and the LiDAR data at the 5270th measurement: (a) Tomoradar normalized power waveform and processed results; and (b) the LiDAR point cloud within the Tomoradar cone (red color) and neighboring ones (blue color).

We observe that the canopy height profile derived from the LiDAR data within the cone of 12 degrees is extremely distinguished from the original result within the cone of six degrees, as Figure 9 presents, which becomes more dispersed and approaches that extracted from the Tomoradar waveform. The improvement of the correlation coefficient from 0 to 0.81 also confirms that it has a higher 
correlation with the Tomoradar result, and the reason is the main lobe leakage of the antenna pattern of Tomoradar system. The antenna pattern is usually strongly peaked along the microwave beam axis, and the spatial dimension is defined by the angular region over which the antenna power pattern is less than $3 \mathrm{~dB}$ below its value at the beam center. However, all antennas have side lobes and part of the received energy comes from outside the main $3 \mathrm{~dB}$ area. Thus, it can lead to some ambiguities due to radiation collected via the side lobes and misinterpreted as radiation in the main lobe, just like the example of the 5270th measurement. The influence of side lobes may be negative or positive, depending on the vegetation structure within the Tomoradar footprint cone, such as the composition, geometry, roughness, position, and so on.

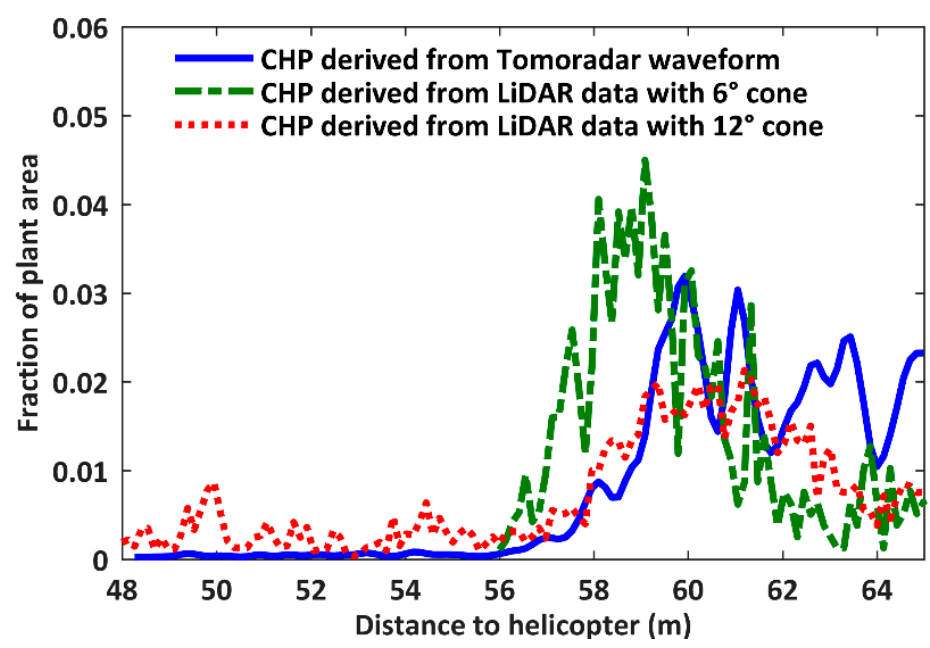

Figure 9. The CHP extracted from the 5270th Tomoradar waveform, and the LiDAR data within the Tomoradar footprint with beam width settings of six degrees and 12 degrees.

To eliminate the influence of side lobes on the extraction of the CHP, the Tomoradar footprint cone is selected as 20 degrees to comprise over $95 \%$ of the energy of the main lobe based on the Tomoradar antenna pattern offered by the antenna manufacturer. Hence, the updated correlation coefficients and histogram of the corresponding proportions are presented in Figure 10.

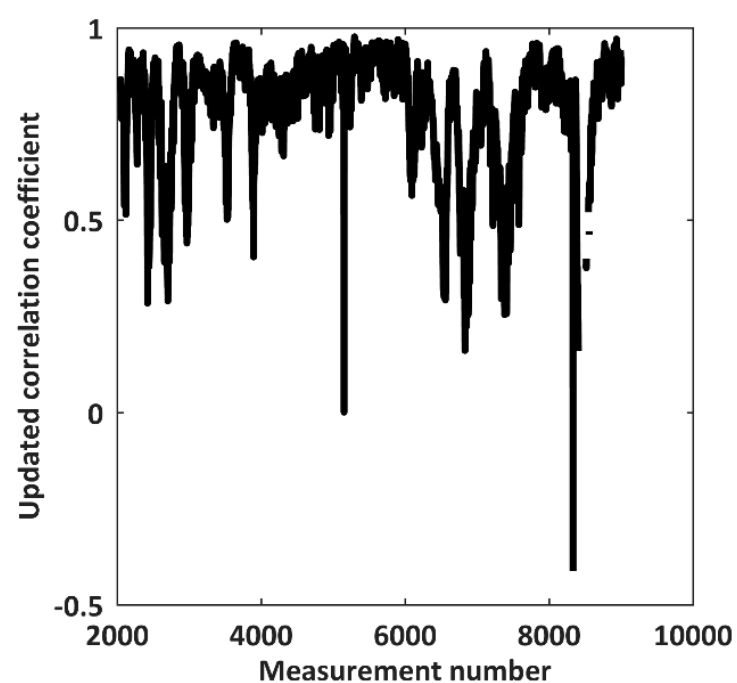

(a)

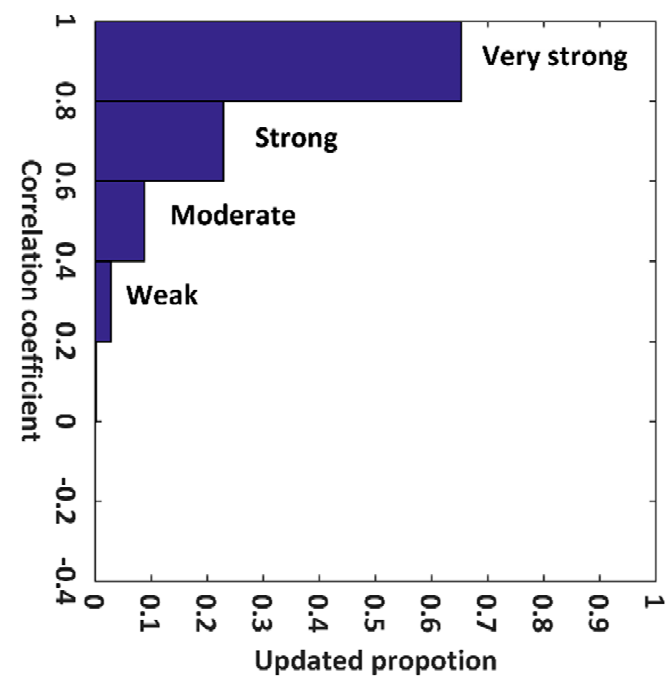

(b)

Figure 10. (a) The updated correlation coefficients of CHP derived from Tomoradar and LiDAR data; and (b) the updated proportions of correlation coefficient, when Tomoradar footprint cone is 20 degrees. 
The updated results in Figure 10a demonstrated that most of the correlation coefficients greatly increase relative to those in Figure 6a. Meanwhile, we noticed that the updated proportions of the correlation coefficients in Figure $9 \mathrm{~b}$ were dependent on the strength of the correlation: very strong $(65.28 \%)$, strong $(22.89 \%)$, moderate $(8.79 \%)$, weak $(2.82 \%)$, and very weak $(0.22 \%)$. An overwhelming majority of the CHPs (96.96\%) derived from Tomoradar waveforms had above-moderate correlation with those from the LiDAR data.

For quantitatively analyzing the differences of the CHP derived from Tomoradar waveforms and the LiDAR data, we calculated the RMSEs of the differences $\left(\delta_{d}\right)$ for all 6766 measurements. Moreover, we presented the illustrations of $\delta_{d}$ and total canopy closures in Figure 11 to reveal the inner connections between them.

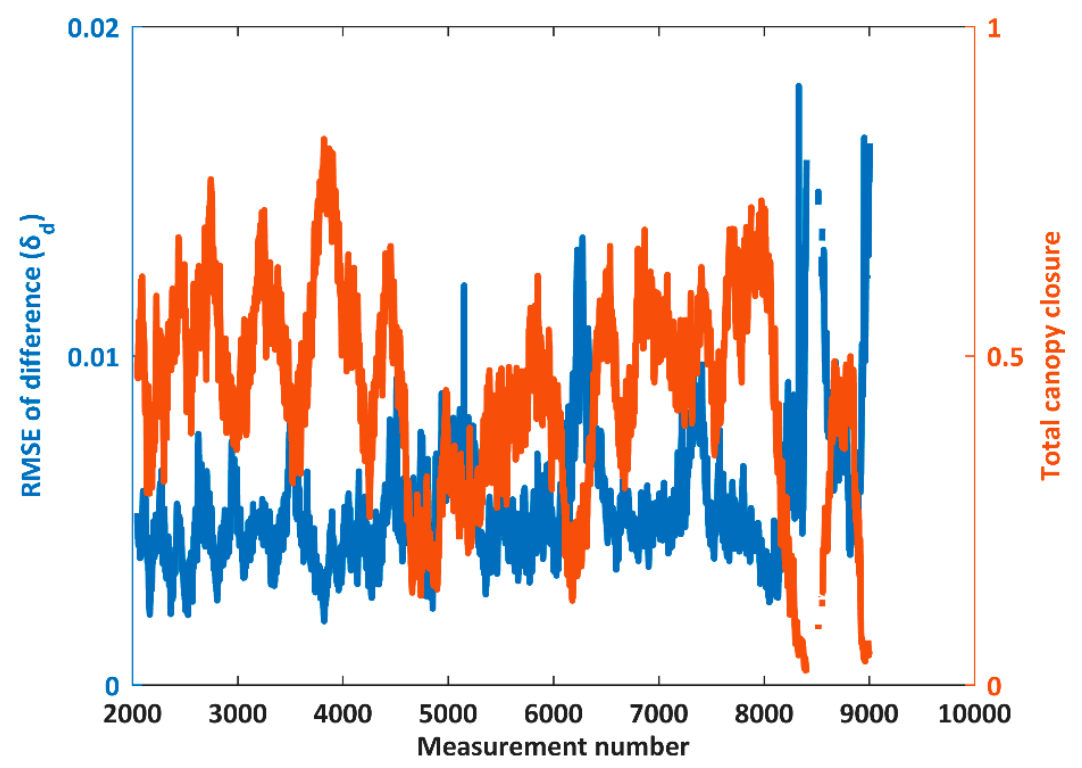

Figure 11. The RMSEs of the difference of CHP derived from Tomoradar waveforms and the LiDAR data (blue color) and the total canopy closure (red color) for all reserved measurements.

About $98 \%$ of the RMSEs of the difference $\left(\delta_{d}\right)$ varied from 0.002 to 0.01 , and the average of the difference $\left(\mu_{d}\right)$ was less than 0.012 . Furthermore, the RMSEs and total canopy closures fluctuated with completely opposite tendencies for all 6766 measurements. This suggested that CHP derived from Tomoradar waveforms were approaching those from the LiDAR data, when the microwave or laser transmits into a relatively open canopy. However, the differences increased if the microwave or laser projected into a denser canopy due to the different penetration capabilities. In Figure 11, the average of $\delta_{d}$ for a canopy with smaller total closure (less than 0.5 ) is 0.0042 , and yet which is 0.0058 for a canopy with larger total closure (larger than 0.5).

\subsection{Linear Regression Results}

The purpose of linear regression is to explore whether the CHPs derived from Tomoradar waveform keep the linear relationships with those from LiDAR data. Taking the 5700th measurement in the stripe of the study area as an example, we presented a scatterplot of the CHP from Tomoradar waveforms versus that from the LiDAR data and the linear regression results in Figure 12. 


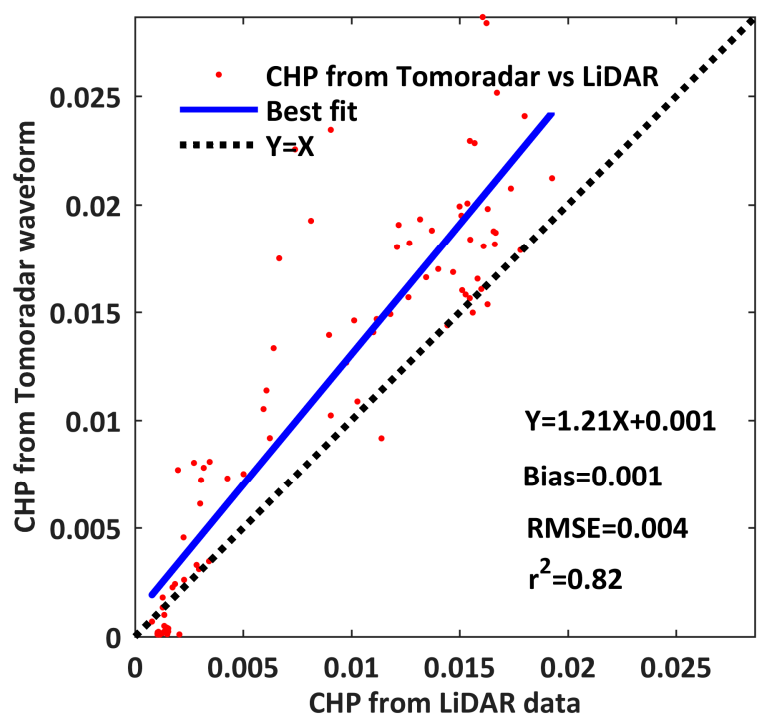

Figure 12. The scatterplot of canopy height profile from Tomoradar data versus that from the LiDAR data canopy height profile (red spots) and best linear fit (blue line).

For obtaining more linear regression results of the CHPs, we computed the coefficients of determination and the RMSEs of residuals for 6766 measurements and provided the corresponding distributions in Figure 13. To investigate the relationship between the regression results and the differences of the CHP, the correlation coefficients and the RMSEs of the differences were also introduced in Figure 13.

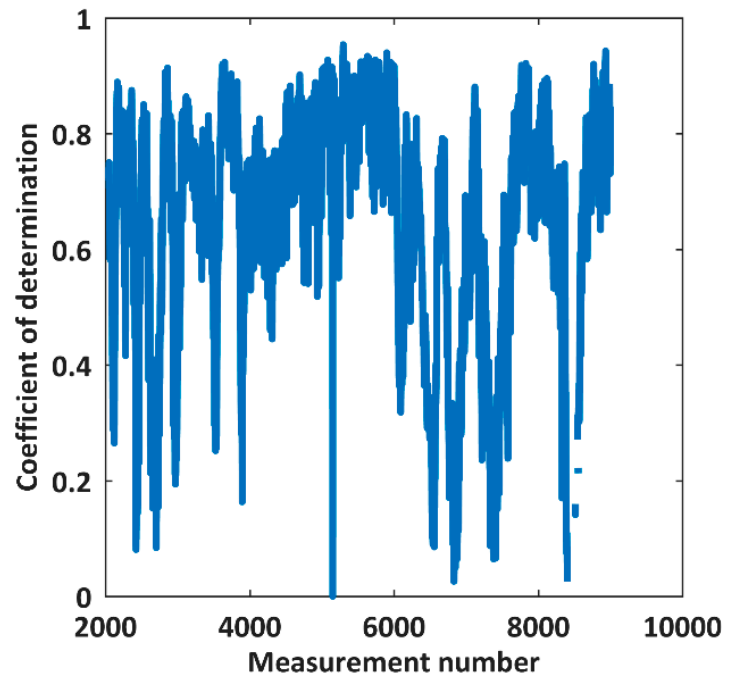

(a)

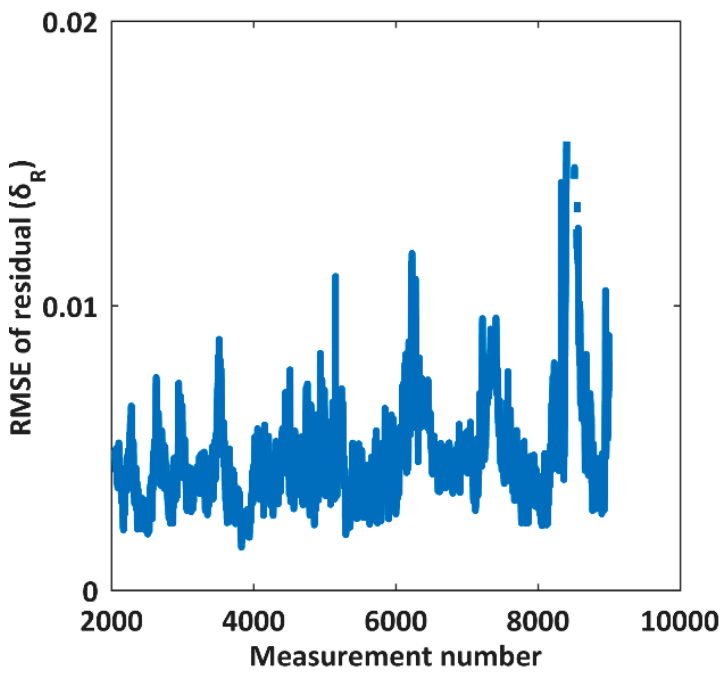

(b)

Figure 13. The distributions of linear regression results for all 6766 measurements: (a) coefficient of determination; and (b) the RMSE of residuals.

Comparing the results of different analyses and linear regressions, we noticed that the coefficients of determination $\left(r^{2}\right)$ and RMSEs of residual $\left(\delta_{R}\right)$ present similar distributions with correlation coefficients $\left(R^{2}\right)$ and RMSE of the differences $\left(\delta_{d}\right)$, individually. However, both $\mathrm{R}^{2}$ and $\delta_{d}$ were greater than $r^{2}$ and $\delta_{R}$, respectively. The comparisons discovered that regression results should be better when the CHPs derived from the Tomoradar waveforms correlate well with those from the LiDAR data. Meanwhile, in Figure 12a, 79.89\% of the coefficients of determination were larger than 0.5, which denoted that most of the relationships between the CHPs from Tomoradar waveforms and those 
from the LiDAR data can be accurately explained by using the linear regression models. Furthermore, $98.89 \%$ of the RMSEs of residuals $\left(\delta_{R}\right)$ ranged from 0.001 to 0.01 , and the averages of the residuals were approximately zero in Figure $12 \mathrm{~b}$.

\section{Conclusions}

In this research, we utilize raw data originating from both Tomoradar and VLP-16 LiDAR on-board the same airborne platform to derive the CHP. Based on the evaluation indicators, including the correlation coefficient, the RMSE of the difference, coefficient of determination, and the RMSE of the residuals of linear regression, the comparisons of the $\mathrm{CHP}$ extracted from these two sensors were thoroughly expounded.

The 6766 effective measurements in one stripe of the study area in Southern Finland were applied in the derivation of the canopy height profile. Through calculation and analysis, we observed that approximately $74.59 \%$ of the measurements had above-moderate correlation strength (a correlation coefficient greater than 0.4 ). However, if the Tomoradar footprint beam width was defined as 20 degrees to contain over $95 \%$ of the transmitting energy of the main lobe, the comparisons of the CHPs derived from Tomoradar and LiDAR data would be notably changed: $96.96 \%$ of the CHPs derived from the Tomoradar waveforms correlated well with those from the LiDAR data (the correlation coefficient is greater than 0.6). Based on the Tomoradar footprint cone, we obtained the following analysis results: (1) about $98 \%$ of the RMSEs of the difference took values from 0.002 to 0.01 , and all averages of the difference $\left(\mu_{d}\right)$ were less than 0.012 ; (2) $79.89 \%$ of the coefficients of determination were larger than 0.5 ; and (3) $98.89 \%$ of the RMSEs of residuals $\left(\delta_{R}\right)$ ranged from 0.001 to 0.01 .

Through investigations in the pape, we can draw some conclusions: (1) Tomoradar waveforms could be employed in the derivation of the CHP, and provide more detailed information inside the canopy than the LiDAR data; (2) in a region with a relatively open canopy, the CHPs from Tomoradar waveforms correlate well and maintain a linear relationship with those from the LiDAR data. However, the differences of the CHP from Tomoradar waveforms and LiDAR data increased in a region with a denser canopy; and (3) because of main lobe leakage, the partial comparisons would be influenced, when the vegetation characters within the outside portion of the main lobe, including density, composition, geometry, and roughness were obviously different from those within the beam width $(-3 \mathrm{~dB})$.

Due to the differences in wavelength and detection means between the Tomoradar and LiDAR, the derived canopy parameters would be diverse to some extent. Except for the CHP, more canopy results, such as percentile height, leaf area index (LAI), biomass, and so on, should be compared in future research. The analysis may provide some references for the application of Tomoradar waveforms and data fusion of LiDAR data in forest investigations. A mechanical scanning version of the Tomoradar might also be investigated for a wider swath with a higher operation efficiency.

Author Contributions: H.Z. and Y.C. wrote the major parts of the paper, and planned the scientific rationale for the Tomoradar measurement and CHP; Z.F., F.L., and C.J. processed the collected data; J.H. reviewed the content and offered substantial improvement for this paper; M.K. designed the practical field test; T.H. and Y.C. design the Tomoradar and performed the field tests, and collected the data; and L.P. helped to composed and reviewed the content for positioning-related context.

Acknowledgments: This research was financially supported by the Academy of Finland projects "Centre of Excellence in Laser Scanning Research (CoE-LaSR) (307362)" and "New laser and spectral field methods for in situ mining and raw material investigations" (project 292648). Additionally, the Chinese Academy of Science (181811KYSB20160113), the Chinese Ministry of Science and Technology (2015DFA70930), and the Shanghai Science and Technology Foundations (18590712600) are acknowledged.

Conflicts of Interest: The authors declare no conflict of interest. 


\section{References}

1. Forest Definition and Extent". United Nations Environment Programme. Available online: https:/ /web.archive.org/web/20100726140947/http:/ /www.unep.org/vitalforest/Report/VFG-01-Forestdefinition-and-extent.pdf (accessed on 10 April 2018).

2. Brokaw, N.; Lent, R. Vertical Structure. In Maintaining Biodiversity in Forest Ecosystems; Hunter, M., Ed.; Cambridge University Press: Boston, MA, USA, 1999; pp. 373-399.

3. Wilson, J.W. Stand structure and light penetration. I. Analysis by point quadrats. J. Appl. Ecol. 1965, 2, 383-390. [CrossRef]

4. Macarthur, R.H.; Horn, H.S. Foliage profile by vertical measurements. Ecology 1969, 50, 802-804. [CrossRef]

5. Aber, J.D. A method for estimating foliage-height profiles in broad-leaved forests. J. Ecol. 1979, 67, 35-40. [CrossRef]

6. Gong, P.; Mei, X.; Biging, G.S.; Zhang, Z. Improvement of an oak canopy model extracted from digital photogrammetry. Photogramm. Eng. Remote Sens. 2002, 63, 919-924.

7. Sheng, Y.; Gong, P.; Biging, G.S. Model-Based Conifer canopy Surface Reconstruction form Photographic Imagery: Overcoming the Occlusion, Foreshortening, and Edge Effects. Photogramm. Eng. Remote Sens. 2003, 69, 249-258. [CrossRef]

8. Song, C.; Dickinson, M.B. Extracting forest canopy structure from spatial information of high resolution optical imagery: tree crown size versus leaf area index. Int. J. Remote Sens. 2008, 29, 5605-5622. [CrossRef]

9. Alonzo, M.; Bookhagen, B.; McFadden, J.P.; Sun, A.; Roberts, D.A. Mapping urban forest leaf area index with airborne lidar using penetration metrics and allometry. Remote Sens. Environ. 2015, 162, 141-153. [CrossRef]

10. Lovell, J.L.; Jupp, D.L.; Culvenor, D.S.; Coops, N.C. Using airborne and ground-based ranging lidar to measure canopy structure in Australian forests. Can. J. Remote Sens. 2003, 29, 607-622. [CrossRef]

11. Coops, N.C.; Hilker, T.; Wulder, M.A.; St-Onge, B.; Newnham, G.; Siggins, A.; Trofymow, J.T. Estimating canopy structure of Douglas-fir forest stands from discrete-return LiDAR. Trees 2007, 21, 295. [CrossRef]

12. Wilkes, P. Assessment of Forest Canopy Vertical Structure with Multi-Scale Remote sensing: From the Plot to the Large Area. Ph.D. Thesis, University of Twente, Enschede, The Netherlands, 2015.

13. Zhang, Z.; Cao, L.; She, G. Estimating Forest Structural Parameters Using Canopy Metrics Derived from Airborne LiDAR Data in Subtropical Forests. Remote Sens. 2017, 9, 940. [CrossRef]

14. Lefsky, M.A.; Cohen, W.B.; Acker, S.A.; Parker, G.G.; Spies, T.A.; Harding, D. Lidar remote sensing of the canopy structure and biophysical properties of Douglas-fir western hemlock forests. Remote Sens. Environ. 1999, 70, 339-361. [CrossRef]

15. Harding, D.J.; Lefsky, M.A.; Parker, G.G.; Blair, J.B. Laser altimeter canopy height profiles: Methods and validation for closed-canopy, broadleaf forests. Remote Sens. Environ. 2001, 76, 283-297. [CrossRef]

16. Allouis, T.; Durrieu, S.; Véga, C.; Couteron, P. Stem volume and above-ground biomass estimation of individual pine trees from LiDAR data: Contribution of full-waveform signals. IEEE J. Sel. Top. Appl. Earth Obse. Remote Sens. 2013, 6, 924-934. [CrossRef]

17. Fieber, K.D.; Davenport, I.J.; Tanase, M.A.; Ferryman, J.M.; Gurney, R.J.; Becerra, V.M.; Hacker, J.M. Validation of Canopy Height Profile methodology for small-footprint full-waveform airborne LiDAR data in a discontinuous canopy environment. ISPRS J. Photogramm. Remote Sens. 2015, 104, 144-157. [CrossRef]

18. Hermosilla, T.; Ruiz, L.A.; Kazakova, A.N.; Coops, N.C.; Moskal, L.M. Estimation of forest structure and canopy fuel parameters from small-footprint full-waveform LiDAR data. Int. J. Wildland Fire 2014, 22, 224-233. [CrossRef]

19. Piermattei, L.; Hollaus, M.; Pfeifer, N.; Chen, Y.; Karjalainen, M.; Hakala, T.; Hyyppä, J.; Wagner, W. Comparing helicopter-borne profiling radar with airborne laser scanner data for forest structure estimation. In Proceedings of the EGU General Assembly Conference Abstracts, Vienna, Austria, 23-28 April 2017; Volume 19, p. 13741.

20. Feng, Z.; Chen, Y.; Hakala, T.; Hyyppä, J. Range calibration of airborne profiling radar used in forest inventory. In Proceedings of the Geoscience and Remote Sensing Symposium (IGARSS), Beijing, China, 10-15 July 2016; pp. 6672-6675.

21. Piermattei, L.; Hollaus, M.; Milenković, M.; Pfeifer, N.; Quast, R.; Chen, Y.; Hakala, T.; Karjalainen, M.; Hyyppä, J.; Wagner, W. An Analysis of Ku-Band Profiling Radar Observations of Boreal Forest. Remote Sens. 2017, 9, 1252. [CrossRef] 
22. Le Toan, T.; Beaudoin, A.; Riom, J.; Guyon, D. Relating forest biomass to SAR Data. IEEE Trans. Geosci. Remote Sens. 1992, 30, 403-411. [CrossRef]

23. Yu, X.; Hyyppä, J.; Karjalainen, M.; Nurminen, K.; Karila, K.; Vastaranta, M.; Kankare, V.; Kaartinen, H.; Holopainen, M.; Honkavaara, E.; Kukko, A.; et al. Comparison of Laser and Stereo Optical, SAR and InSAR Point Clouds from Air- and Space-Borne Sources in the Retrieval of Forest Inventory Attributes. Remote Sens. 2015, 7, 15933-15954. [CrossRef]

24. Chen, Y.; Hakala, T.; Karjalainen, M.; Feng, Z.; Tang, J.; Litkey, P.; Kukko, A.; Jaakkola, A.; Hyyppä, J. UAV-Borne Profiling Radar for Forest Research. Remote Sens. 2017, 9, 58. [CrossRef]

25. User's Manual and Programming Guide VLP-16 Velodyne LiDAR. Available online: http:/ / velodynelidar. com/docs/manuals / 63-9243\%20Rev\%20B\%20User\%20Manual\%20and\%20Programming\%20Guide, VLP16.pdf (accessed on 10 April 2018).

26. Parrish, C.E.; Jeong, I.; Nowak, R.D.; Smith, R.B. Empirical comparison of full-waveform lidar algorithms. Photogramm. Eng. Remote Sens. 2011, 77, 825-838. [CrossRef]

27. Morsdorf, F.; Kötz, B.; Meier, E.; Itten, K.I.; Allgöwer, B. Estimation of LAI and fractional cover from small footprint airborne laser scanning data based on gap fraction. Remote Sens. Environ. 2006, 104, 50-61. [CrossRef]

28. Feng, Z.; Chen, Y.; Hyyppä, J.; Hakala, T.; Zhou, H.; Wang, Y.; Karjalainen, M. Estimating Ground Level and Canopy Top Elevation with Airborne Microwave Profiling Radar. IEEE Trans. Geosci. Remote Sens. 2018, 99, 1-12. [CrossRef]

(C) 2018 by the authors. Licensee MDPI, Basel, Switzerland. This article is an open access article distributed under the terms and conditions of the Creative Commons Attribution (CC BY) license (http://creativecommons.org/licenses/by/4.0/). 